\title{
Sobre os Sistemas de Referência Celeste
}

\author{
On The Celestial Reference Systems
}

\author{
V.A.F. Martin* e P.C.R. Poppe ${ }^{\dagger}$ \\ Departamento de Física - UEFS \\ Campus Universitário $\mathrm{Km} 03 \mathrm{BR} 116$ \\ Observatório Astrônomico Antares - UEFS \\ Feira de Santana - BA - 44031-460
}

\begin{abstract}
Apresentamos neste trabalho algumas discussões sobre os Sistemas de Referência utilizados em Astronomia. Claramente, não é possível esgotar todo este assunto num único texto, mas esperamos, contudo, que o presente material possa ser apreciado nos cursos de Introdução à Astronomia, que estão cada vez mais presentes nas atuais propostas curriculares das graduações de Física. As discussões pertinentes às "Bases de Referência Celeste" (Celestial Reference Frame), serão apresentadas em um outro trabalho.
\end{abstract}

Palavras-chaves: Astronomia, Sistemas de Referência, Currículos de Graduação.

Some discussions about Celestial Reference Systems in Astronomy are exposed in this paper. In spite of exist a vast literature for this purpose, we hope that the present material could be usefully employed in introductory courses of Astronomy, which are usually offered in the undergraduate curriculum of physics. The discussions about the "Celestial Reference Frame" will be treated in another paper.

Key-words: Astronomy, Celestial Reference frame, Undergraduation Programs.

\section{INTRODUÇÃO}

\section{SISTEMAS DE REFERÊNCIA}

Ao longo do curso introdutório de Física I (Mecânica), aprendemos que um corpo "A" está em movimento relativo a um corpo "B", quando sua posição, medida com relação ao segundo corpo, varia no tempo. Quando sua posição relativa não varia com o tempo, dizemos que o mesmo está em repouso relativo. Portanto, repouso e movimento são conceitos relativos, isto é, dependem da escolha do corpo que serve como referência. Podemos exemplificar dizendo que tanto uma árvore quanto uma casa estão em repouso relativo à Terra, porém, em movimento relativo ao Sol, ou ainda, dizer que um trem ao passar por uma estação encontra-se em movimento relativo à mesma. Todavia, um passageiro no interior do trem pode, simplesmente, afirmar que é a estação ferroviária que está em movimento relativo ao trem.

$\mathrm{Na}$ verdade, procuramos verificar com esta abreviada discussão que, para descrever o movimento, o observador deve definir um "sistema de referência" ou "referencial" em relação ao qual o movimento será analisado. Desse modo, e não sendo diferente tal abordagem na Astronomia, discutiremos neste trabalho (e também em sua continuação: próximo boletim) que o estudo dos movimentos e das posições dos corpos celestes necessitam igualmente de definições e construções precisas dos chamados Sistemas de Referência [1].

\footnotetext{
*Endereço Eletrônico: vmartin@uefs.br

$\dagger$ Endereço Eletrônico: poppe@uefs.br
}

Historicamente, coube a Galileu Galilei (1564-1642) o mérito de ter sido o primeiro a demonstrar a importância dos sistemas de referência na formulação das leis que regem a descrição dos fenômenos físicos, estabelecendo desse modo, uma relação mensurável entre leis e grandezas físicas.

Um sistema de referência pode ser descrito, de maneira muito simplificada, por meio de um terno de eixos cartesianos ortogonais $(O ; x, y, z)$ solidários com o observador que considera, por exemplo, o movimento de pontos materiais de um dado sistema físico. Contudo, um sistema de eixos de coordenadas é uma noção abstrata e que não é acessível a todo tempo, de tal forma que é preciso materializar o sistema de referência por meio de um certo número de pontos de referência (parâmetros), de modo a poder determinar as coordenadas e eventualmente os movimentos.

$\mathrm{Na}$ Astronomia, um sistema de referência (dito fundamental) é materializado por meio de um conjunto de parâmetros adotados por convenção internacional (isto é, posição e movimento próprio), a partir de estrelas convenientemente selecionadas e denominadas estrelas fundamentais [2], que presentes num catálogo fundamental, constituem a materialização para o sistema de referência.

Esta materialização é conhecida como "reference frame" (em inglês) ou "repère de référence" (em francês). Para o nosso caso, traduziremos como "base de referência". Esta distinção entre sistema de referência (o conceito teórico) e base de referência (a materialização através de objetos astronômicos) foi devidamente formalizada por Kovalevsky e Mueller em 1989 [3] e corresponde a diferença atual entre "reference system" e "reference frame". 


\section{A. Aspectos Cinemáticos e Dinâmicos}

Segundo Kovalevsky e Mueller [3], podemos distinguir o sistema de referência dinâmico do sistema de referência cinemático. Para o primeiro, o sistema está baseado no estudo dinâmico dos corpos celestes, isto é, na resolução das equações diferenciais de seus movimentos, seja no campo da Mecânica Newtoniana ou da Mecânica Relativística. Para os corpos do Sistema Solar, veremos que o primeiro caso pode ser considerado como o de ordem zero do segundo.

No que tange ao sistema de referência cinemático, este está baseado no estudo estatístico dos movimentos de corpos longínquos, estrelas distantes e sobretudo, galáxias e quasares, do ponto de vista puramente cinemático, isto é, sem se referir às causas desses movimentos.

No que segue, apresentaremos de maneira bastante resumida alguns passos necessários para a construção de tais sistemas. O leitor encontrará em Kovalevsky [4] uma ampla e completa discussão.

\section{a) Concepção (ou definição ideal)}

A idéia intuitiva de que um sistema de referência celeste deva ser um sistema sem rotação, não é muito fácil de ser axiomatizada, apesar de existir maneiras diferentes de expressar tal idéia, quer seja dentro de uma abordagem dinâmica ou cinemática. Para o primeiro caso, em relação a um sistema celeste ideal, os astros movem-se de tal maneira que as equações que descrevem o movimento não possuem termos de aceleração (de rotação ou de translação não uniforme). Claramente, dentro de um universo regido pela Relatividade Geral, esta definição newtoniana só pode ser aplicada localmente.

Para o segundo caso, o sistema ideal é definido pela ausência de rotação em relação a um conjunto de pontos de referência que não apresentam movimento próprio. O significado físico desta proposição, entretanto, é duvidosa. Em contrapartida, sua aplicação ao universo real é possível, dentro de uma certa aproximação, e pode ter um caráter não local.

Em tempo, alertamos que o emprego do termo "sistema inercial" para estes sistemas não é próprio. Convém falar, estritamente, de sistemas dinâmicos dentro de um universo newtoniano.

\section{b) Estrutura Física}

Dentro de uma construção cinemática, temos que as galáxias e os quasares são os melhores candidatos para servir de referência a uma tal definição. Já para aquela dinâmica, o estudo dinâmico dos objetos do Sistema Solar, isto é, a resolução das equações diferenciais de seus movimentos, seja na Mecânica Clássica ou na Relativística, representa a base para tal definição. O movimento da Lua ou de um conjunto de satélites artificiais podem igualmente ser utilizados.

\section{c) Modelização da Estrutura}

Para a definição cinemática, as galáxias e os quasares estão suficientemente distantes $\left(10^{8}\right.$ anos luz ou mais), de modo que representam um excelente modelo de pontos fixos no universo. Podemos mesmo escolher um para definir de maneira convencional a origem de um sistema de coordenadas. Por outro lado, mesmo que os seus movimentos próprios fossem da ordem de grandeza de suas velocidades de afastamento, eles dificilmente seriam observados, isto é, seriam inferiores a $10^{-5}$ segundos por ano.

Para a definição dinâmica, os valores adotados para um certo número de parâmetros fundamentais relativos a dinâmica do Sistema Solar e a junção entre sistemas celeste e terrestre intermediários, constituem o sistema IAU de constantes astronômicas.

\section{d) Materialização do Sistema}

Para a definição dinâmica, a realização de um sistema é assegurada por uma teoria numérica dos movimentos dos planetas, em particular, o da Terra. As coordenadas das estrelas de referência são determinadas em relação às posições teóricas dos corpos do Sistema Solar, graças às observações relativas de estrelas e do Sol (catálogos absolutos) ou de planetas. Isto define praticamente a base de referência [5] com os catálogos fundamentais $(F K 4,5)$, por exemplo. Notemos entretanto, que na prática estes catálogos constituem de certa forma uma extensão do sistema de referência, onde nem todas as estrelas estão diretamente ligadas ao Sol ou aos planetas. As efemérides constituem uma das materializações da base de referência definida dinamicamente.

Para a definição cinemática, as coordenadas equatoriais de quasares determinadas por VLBI, na ordem do mas, materializam os sistemas de referência cinemático [6]. Notemos, entretanto, que apesar do antigo sistema convencional de referência $(F K 5)$ e sua realização se apoiarem na dinâmica do Sistema Solar, eles utilizam, igualmente, resultados provenientes da cinemática de Galáxia [7].

\section{e) Extensão da base convencional}

O número de pontos de referência cuja a posição é determinada pode ser insuficiente para todas as aplicações práticas, ou ainda, estes pontos podem não ser acessíveis a outros tipos de instrumentos. A extensão natural da base de referência dinâmica ou cinemática consiste em determinar as posições e os movimentos próprios de um grande número de estrelas que não pertencem ao catálogo fundamental em relação ao sistema materializado por ele.

Em relação a definição dinâmica, o problema principal que se coloca é de se estabelecer uma conexão entre as posições e os movimentos próprios das estrelas que não possuem emissão rádio no sistema definido pelas rádio-galáxias. Sem uma tal conexão, o sistema de referência cinemático não será mais utilizável em astronomia galáctica. É desejável, todavia, de não perder, dentro desta conexão, a precisão intrínseca da base de referência VLBI. O estágio atual consiste em conectar a base VLBI com aquela definida pelas observações HIPPARCOS.

\section{B. Mecânica Newtoniana}

A Mecânica Newtoniana separa as coordenadas espaciais da coordenada temporal, introduzindo para as primeiras a 
noção de sistema de referência inercial e para a segunda a noção de tempo uniforme. Nesta, o postulado da relatividade galileana diz que duas experiências idênticas realizadas em dois referenciais em translação uniforme, um em relação ao outro, fornecem resultados idênticos. Portanto, podemos concluir que as leis da Física Clássica são invariantes em relação às leis de transformação de Galileu:

$$
x^{\prime}=x+v_{x} t, \quad y^{\prime}=y+v_{y} t, \quad z^{\prime}=z+v_{z} t, \quad t^{\prime}=t,
$$

para um mesmo evento expresso em dois referenciais $r$ e $r^{\prime}$, onde este último se desloca com uma velocidade $v$ (de componentes $v_{x}, v_{y}$ e $v_{z}$ ) constante em relação ao primeiro. Estamos admitindo os dois observadores usando o mesmo tempo, isto é, admitimos as medidas de tempo independentes do movimento do observador. Isso parece muito razoável, embora seja uma hipótese passível de ser contrariada pela experiência.

\section{Mecânica Relativística}

Em 1905, o físico e matemático germano-norte-americano Albert Einstein (1879-1955), propôs um princípio de relatividade especial afirmando que "todas as leis da natureza (não apenas as da dinâmica) devem ser as mesmas para todos os observadores inerciais que se movem, um em relação ao outro, com velocidades relativas constantes".

Einstein admitiu a velocidade da luz como um invariante físico, tendo o mesmo valor para todos os observadores. Admitida essa hipótese, a transformação de Galileu não pode ser correta. Em particular, a equação $t^{\prime}=t$ não pode ser mais correta. Como a velocidade é a distância dividida pelo tempo, pode ser que tenhamos de ajustar o tempo bem como a distância para que o quociente dos dois permaneça com o mesmo valor para observadores em movimento relativo, como acontece no caso da velocidade da luz. Em outras palavras, o intervalo de tempo entre dois acontecimentos não é o mesmo para dois observadores em movimento retilíneo. Desse modo, devemos substituir a transformação de Galileu por outra, de modo que a velocidade da luz seja invariante.

A Relatividade Especial introduz o conceito de espaçotempo, pelo qual compreende-se que o conceito de espaço não pode ser dissociado da noção de tempo. O tempo é local e varia de um sistema a outro. Define-se ainda "sistemas inerciais", que, através da convenção da soma de Einstein, a métrica pode ser escrita como:

$$
d s^{2}=-\left(d x^{0}\right)^{2}+d x^{i} d x^{i}
$$

onde $x_{0}=c t$ é a coordenada temporal e os $x_{i}(i=1,2,3)$ as coordenadas espaciais; $c$ é a velocidade da luz. Claramente, através das Transformações de Lorentz, podemos passar de um sistema inercial a outro.

Para a Relatividade Geral, por outro lado, não existe a noção de um "sistema de referência privilegiado". Podemos simplesmente dizer que certos sistemas são mais apropriados que outros para certos problemas. Aliás, não existe mais o conceito de sistemas de referência universal, mas sim, de sistemas locais.

\section{SISTEMA SOLAR E A HIERARQUIA DOS SISTEMAS DE REFERENCIA}

No interior do Sistema Solar, e com ajuda da equação (2), podemos utilizar sistemas de referência cujas métricas são desenvolvidas em função do pequeno parâmetro $v / c$, isto é:

$$
d s^{2}=g_{00}\left(d x^{0}\right)^{2}+g_{0 i} d x^{0} d x^{i}+g_{i j} d x^{i} d x^{j},
$$

com

$$
g_{00}=-\left(1+h_{00}\right) ; \quad g_{0 i}=-h_{0 i} ; \quad g_{i j}=\delta_{i j}-h_{i j}
$$

onde a função $\delta_{i j}=0$ para $i \neq j$ e $\delta_{i j}=1$ para $i=j$. A velocidade $v$ é definida como:

$$
v^{2}=\sum_{i=1}^{3}\left(\frac{d x^{i}}{d t}\right)^{2}
$$

Os $h_{00}, h_{0 i}$ e $h_{i j}$, são desenvolvimentos em função do pequeno parâmetro $v / c$ sem o termo de ordem zero: $h_{00}$ e $h_{i j}$ comportam somente potências pares do pequeno parâmetro e os $h_{0 i}$ as potências impares. Na ordem zero de $v / c$, estas métricas se reduzem à métrica da relatividade restrita, os sistemas de referência aos de sistemas inerciais e os movimentos dos corpos as soluções fornecidas pela Mecânica Newtoniana.

De acordo com o russo Viktor Aleksandrovich Brumberg (1991), podemos estabelecer no interior do nosso Sistema Solar, uma espécie de hierarquia [8] de sistemas de referência (SR), isto é:

(i) SR Baricêntrico (SRB): centrado no baricentro do Sistema Solar;

(ii) SR Heliocêntrico (SRH): centrado no Sol;

(iii) SR Terra-Lua (SRTL): centrado no baricentro do sistema Terra-Lua;

(iv) SR Geocêntrico (SRG): centrado no centro de massa da Terra;

(v) SR Topocêntrico (SRT): com origem num ponto situado na superfície da Terra.

De acordo com a resolução A4 IAU de 1991 (Buenos Aires), o tempo coordenado $t=x_{0} / c$ do SRB é o TCB (tempo coordenado baricêntrico); não obstante, o TDB (tempo dinâmico baricêntrico) que difere do TCB por um termo proporcional ao tempo, foi igualmente utilizado. Inúmeras interpretações (o que ocasionaram várias confusões) sobre o dueto sistemas de referência e escalas de tempo, conduziram à resoluções posteriores C7, B6 e B1 nas IAU's de 1994 (Hague, Holanda), 1997 (Kyoto, Japão) e 2000 (Manchester, Inglaterra), respectivamente. Sugerimos ao leitor, para um discussão das resoluções IAU sobre sistema de referência e escalas de tempo, a recente publicação feita por Brumberg e Groten [9].

Para o SRB, uma vez desprezada a influência da Galáxia e tomando as hipóteses da Relatividade Geral, podemos escrever a métrica para este sistema como:

$$
\begin{aligned}
h_{00} & =-\frac{2 U}{c^{2}}-\frac{2 W}{c^{4}}+\ldots, \\
h_{0 i} & =-\frac{4 U^{i}}{c^{3}}+\ldots, \\
h_{i j} & =-\frac{2 \delta_{i j} U}{c^{2}}+\ldots,
\end{aligned}
$$


onde $U$ é o potencial newtoniano criado por todos os corpos do Sistema Solar e $U^{i}$ o potencial vetor. Supondo corpos pontuais, podemos escrever simplesmente:

$$
U=\sum_{A} \frac{G M_{A}}{r_{A}} \quad \text { e } \quad U^{i}=\sum_{A} \frac{G M_{A}}{r_{A}} v_{A}^{i},
$$

onde os somatórios são estendidos a todos os corpos do Sistema Solar; $M_{A}$ é a massa do corpo $A$ e $x_{A}^{i}$ suas coordenadas espaciais. Nestas equações, $r_{A}$ é definido por:

$$
\begin{aligned}
\left(r_{A}\right)^{2} & =\sum_{i}\left(x^{i}-x_{A}^{i}\right)^{2} \\
v_{A}^{i} & =\frac{d x_{A}^{i}}{d t}
\end{aligned}
$$

Encontraremos as expressões mais completas de $U$ e $U^{i}$, bem como a expressão de $W$ (potencial da força de gravidade) em Brumberg e Kopejkin [10].

\section{TRANSFORMAÇÃO DE COORDENADAS ENTRE SR}

Vimos ao longo das seções anteriores que o movimento é um conceito relativo e que deve sempre ser referido a um referencial específico, escolhido pelo observador. Desde que diferentes observadores possam usar diferentes referenciais, é importante saber como relacionar suas observações. De acordo com Brumberg [11], por exemplo, podemos levar as coordenadas harmônicas presentes no SRB, para as coordenadas harmônicas $w^{0}=c u$ e $w^{i}$ no SRG, através das seguintes relações de transformação:

$$
\begin{aligned}
u= & t-\frac{1}{c^{2}}\left[S+v_{E}^{k}\left(x^{k}-x_{E}^{k}\right)\right]+\ldots \\
w^{i}= & x^{i}-x_{E}^{i}+\frac{1}{c^{2}}\left[\left(\frac{1}{2} v_{E}^{i} v_{E}^{k}+F^{i k}+D^{i k}\right)\left(x^{k}-x_{E}^{k}\right)\right. \\
& \left.+D^{i j k}\left(x^{j}-x_{E}^{j}\right)\left(x^{k}-x_{E}^{k}\right)\right]+\ldots
\end{aligned}
$$

onde os $x_{E}^{k}$ designam as coordenadas espaciais SRB da Terra, $v_{E}^{k}$ suas derivadas em relação a $t ; S, F^{i k}, D^{i k}, D^{i j k}$ são funções de $t$ calculáveis a partir das coordenadas espaciais da Terra, dos planetas e das massas; os $F^{i k}$ são anti-simétricos em $i$ e $k$. O termo relativista principal da relação ligando as coordenadas espaciais pode ser colocado na forma:

$$
\frac{1}{c^{2}} F^{i k}\left(x^{k}-x_{E}^{k}\right)=\frac{1}{c^{2}}\left[\left(\vec{x}-\vec{x}_{E}\right) \times \vec{F}\right]^{i},
$$

onde $\vec{x}$ e $\vec{x}_{E}$ são os vetores de $\mathbb{R}^{3}$ de componentes $x^{i}$ e $x_{E}^{i}$; $\vec{F}$ é o vetor de componentes:

$$
F^{i}=\frac{1}{2} \epsilon_{i j k} F^{j k}
$$

com $\epsilon_{i j k}=0$, se dois dos índices forem iguais, $\epsilon_{123}=1 \mathrm{e}$ $\epsilon_{i j k}=-\epsilon_{j i k}=-\epsilon_{i k j}$.

Em primeira aproximação, $c^{-2} \vec{F}$ é um vetor perpendicular à eclíptica cuja intensidade, de acordo com De Sitter [12], vale:

$$
p_{g} t=\frac{3 k^{2}}{2 c^{2}} \frac{n_{s}}{a_{s}} t
$$

onde $k$ é a constante de Gauss, $n_{s}$ e $a_{s}$ o movimento médio e o semi-eixo da órbita terrestre, respectivamente; $p_{g}$ é a precessão geodésica. Os eixos espaciais do SRG aparecem, portanto, em rotação uniforme em relação aos eixos espaciais do SBR com velocidade angular $p_{g}$.

De maneira mais rigorosa em Brumberg et al. 1991 [13], as componentes de $c^{-2} \vec{F}$ num sistema espacial definido pela eclíptica e pelo equinócio médio de J2000, se decompõem numa parte secular e numa periódica. A precessão geodésica é então definida como o coeficiente do termo em $t$, onde $t$ é contado a partir de J2000, e da componente $c^{-2} F^{3}$, perpendicular à eclíptica. Seu valor é de $1^{\prime \prime}, 9199$ por século juliano. Podemos encontrar em [10] a expressão completa de $c^{-2} F^{3}$. A amplitude dos termos periódicos é maior que $0^{\prime \prime}, 000153$.

\section{CONCLUSÕES}

Podemos dizer que um dos objetivos da Astronomia Fundamental é o de materializar sistemas de referência que se aproximem, tanto quanto possível, de um sistema inercial, isto é, sem acelerações ou rotações residuais. Isto significa dizer que, inevitavelmente, aspectos clássicos e relativísticos serão constantemente chamados para discussão. Apesar desta importante inserção, ainda verificamos uma tímida abordagem (além de outras) por parte dos livros textos que são geralmente empregados nos cursos de graduação e de pós-graduação.

Em virtude desta constatação, procuramos discutir dentro deste pequeno artigo alguns aspectos sobre sistemas de referência e relatividade, objetivando provocar uma série de discussões estritamente ligadas a inserção da Astrometria (com uma nova roupagem) nos curriculas de graduação dos cursos de Física, através de um enfoque que esteja presente nas diversas disciplinas trabalhadas durante o período de formação do alunado. Esta idéia é fruto de uma ampla discussão sobre mecanismos de aprimoramento da qualidade do ensino de Física na graduação na UEFS. Em particular, a motivação deste trabalho está presente na disciplina Introdução à Astronomia ( $5^{\circ}$ Semestre), obrigatória para os cursos de licenciatura e bacharelado.
[1] Reservaremos para um outro trabalho toda a discussão pertinente à materialização dos sistemas de referência utilizados em Astronomia.

[2] Estrelas cujas posições e movimentos próprios são bem de- terminados, e que são utilizáveis em um catálogo fundamental.

[3] J. Kovalevsky, I.I. Mueller; Introduction, in: Reference Frames in Astronomy and Geophysics; J. Kovalevsky, I.I. 
Muller and B. Kolaczek (eds.), Kluwer, Dordrecht, 1-12 (1989).

[4] J. Kovalevsky; Systèmes de Référence Terrestre et Célestes, Bulletin Astronomique du Observatoire Royal de Belgique, X (2) 87 (1985).

[5] W. Fricke; Where is the equinox, in: Dynamics of the Solar System, IAU Symposium 81, R.L. Duncomnbe (ed.), 133142 (1979).

[6] W.M. Folkner, P. Charlot, M.H. Finger, J.G. Williams, O.J. Sovers, X.X. Newhall, E.M.Jr. Standish; , Determination of the extragalactic-planetary frame tie from joint analysis of radio interferometric and lunar laser ranging measurements, A \& A, 287, (1), 279 (1994).

[7] W. Fricke; Definition and practical realization of the reference frame in the FK5 - The role of planetary dynamics and stellar kinematics in the definition, in: Reference Coordinate Systems for Earth Dynamics, E.M. Gaposchkin and B. Kolaczek (eds.), IAU Colloquium 56, 331-340 (1981).

[8] V.A. Brumberg; Relativistic Hierarchy of Reference Systems and Time Scales, in Proceedings of the 127th Collo- quium of IAU, J.A. Hughes, C.A. Smith and G.H. Kaplan (eds.), USNO, Washington D.C., 36 (1991).

[9] V.A. Brumberg, E. Groten; AU resolutions on reference systems and time scales in practice, A \& A, 367 1070-1077 (2001).

[10] V.A. Brumberg, S.M. Kopejkin; Relativistic Theory of Celestial Reference Frames, in Reference Frames in Astronomy and Geophysics, J. Kovalevsky, I.I. Mueller and B. Kozaczek (eds.), Kluwer, Dordrecht, 154115 (1989).

[11] V.A. Brumberg; Essential Relativistic Celestial Mechanics, Adam Hilger, Bristol, 271 (1991).

[12] W. De Sitter; On the System of Astronomical Constants, Bulletin of the Astronomical Institute of the Netherlands, 8213 (1938).

[13] V.A. Brumberg, P. Bretagnon, G. Francou; Analytical Algorithms of Relativistic Reduction of Astronomical Observations, in Journées, Système de Référence Spatio-Temporels, N. Capitaine (ed.), Observatoire de Paris, 141 (1991). 